\title{
Does perceptual confidence facilitate cognitive control?
}

\author{
Ai Koizumi • Brian Maniscalco • Hakwan Lau
}

Published online: 4 March 2015

(C) The Psychonomic Society, Inc. 2015

\begin{abstract}
Our visual perception is typically accompanied by a sense of subjective confidence. Since perceptual confidence is related to prefrontal activity, higher perceptual confidence may enhance cognitive control functions. To examine this interaction, we developed a novel method to selectively manipulate perceptual confidence while keeping stimulus discrimination accuracy constant. In a behavioral experiment, grating stimuli with different orientations were presented as go/no-go signals. Surprisingly, the results showed that confidence in visual discrimination of the signals on its own did not facilitate response inhibition, since when participants were presented with stimuli that yielded higher confidence, they were no better at performing a go/no-go task. These results were replicated with different (dot motion) stimuli, ruling out alternative explanations based on stimulus idiosyncrasy. In a different experiment, when the grating stimuli were presented as cues for task set preparation, we found that higher perceptual confidence also did not enhance task set preparation efficiency. This result was again replicated with dot motion stimuli. Since confidence may relate to perceptual awareness
\end{abstract}

A. Koizumi $(\bowtie) \cdot$ B. Maniscalco $\cdot$ H. Lau

Department of Psychology, Columbia University, 406 Schermerhorn

Hall, 1190 Amsterdam Ave MC 5501, New York, NY 10027, USA

e-mail: bellkoizumi@gmail.com

A. Koizumi

Department of Psychology, University of Tokyo, Tokyo, Japan

A. Koizumi

US-Japan Brain Research Cooperation Program, Okazaki, Japan

B. Maniscalco

National Institute of Neurological Disorders and Stroke, National Institutes of Health, Bethesda, MD, USA

H. Lau

Department of Psychology, University of California,

Los Angeles, CA, USA
(Peirce \& Jastrow, 1885), our findings may put current dominant theories in question, since these theories often suppose the critical involvement of consciousness in cognitive control. As a proof of concept, our method may also provide a new and powerful way to examine other functions of consciousness in future studies.

Keywords Perceptual confidence $\cdot$ Visual perception . Confidence $\cdot$ Cognitive control $\cdot$ Signal detection theory

In performing simple perceptual decisions (e.g., target discrimination), one may feel more confident in some trials than in others. Although such confidence often reflects perceptual accuracy, recent findings have suggested that the two may dissociate (Del Cul, Dehaene, Reyes, Bravo, \& Slachevsky, 2009; Lau \& Passingham, 2006, 2007; Rounis, Maniscalco, Rothwell, Passingham, \& Lau, 2010). What functional role does perceptual confidence serve? Despite increasing interest in this topic (Fleming \& Dolan, 2012; Lau, 2009), this question remains unanswered, perhaps partly because it is a challenge to test the effects of perceptual confidence per se, independently of the effects of perceptual capacity; when one manipulates confidence, perceptual capacity typically also changes.

Here we developed a novel method to dissociate the effects of confidence from those of perceptual capacity, on the basis of a recent empirical finding (Maniscalco \& Lau, 2012; Zylberberg, Barttfeld, \& Sigman, 2012). In psychophysics experiments, the observer typically has to choose a unique perceptual interpretation of a stimulus, even though the available sensory evidence may be ambiguous. We can distinguish the sources of evidence supporting the correct stimulus identification response (positive evidence, PE) from the sources of evidence that support the alternative response (negative 
evidence, NE). Assuming that one chooses the correct response on a trial, one's confidence should be influenced by both PE and NE; specifically, confidence, like perceptual capacity $\left(d^{\prime}\right)$, should depend on the difference between the two, with higher PE and lower NE both driving higher confidence. However, Zylberberg et al. found this to be untrue, in that confidence seemed to be driven only by the magnitude of PE in their study. Specifically, in their study, participants made a simple perceptual decision (on dot motion direction or luminosity) and then rated their perceptual confidence. The confidence rating was mostly explained by the sensory evidence confirming the perceptual decision (i.e., PE; but see the Exp. 1A Method for the terminology difference between their study and ours). This suggests that perceptual confidence arises from some distinctive perceptual processing that is more stochastic than that behind the perceptual decision, which may make use of both PE and NE. This phenomenon entails the theoretical possibility that, by suitable control of the stimulus properties, one should be able to create stimulus sets that yield similar $d$ 's but different levels of confidence. Specifically, one should be able to increase perceptual confidence by increasing both the PE and NE in a stimulus, while keeping $d^{\prime}$ constant (see Figs. 1A and 2A below).

Importantly, perceptual certainty may also be linked to some forms of visual awareness. For example, patients with lesions to V1 may develop blindsight (Weiskrantz, 2009), in which conscious visual experiences are abolished, even though such patients have above-chance perceptual accuracy.
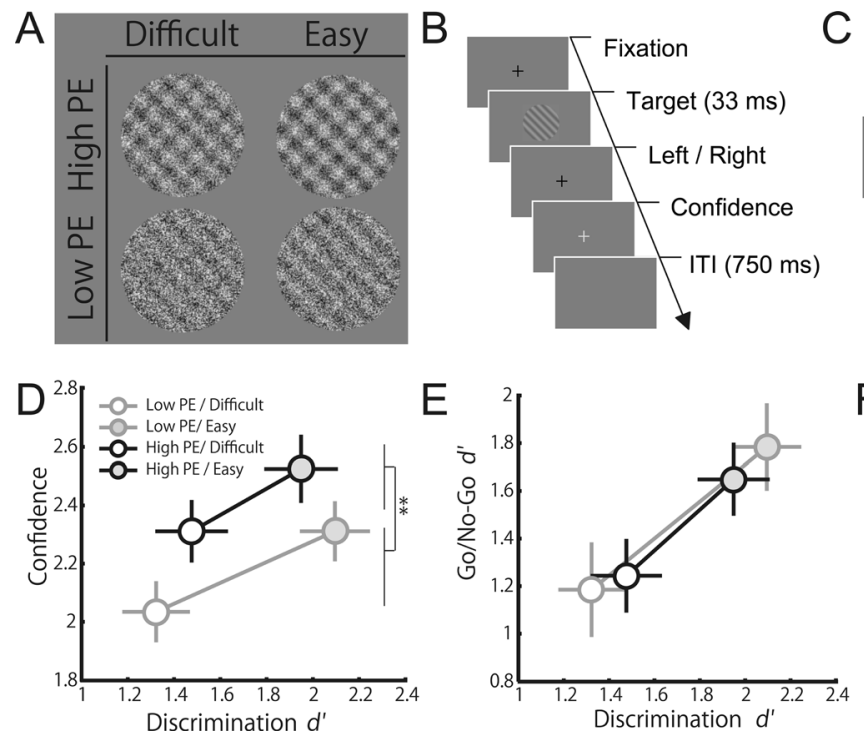

Fig. 1 (A) Task stimuli in Experiments 1A and 2A. Each stimulus consisted of positive evidence (PE; grating tilted toward the left or right), negative evidence (NE; grating tilted in the opposite direction from PE), and noise. The stimuli had either low or high levels of PE, with two difficulty levels. The contrast levels of evidence were iterated for each participant (see the Method section). (B) Trial sequence of the discrimination blocks in Experiments 1A and 2A. (C) Trial sequence of the go/no-go block in Experiment 1A. Stimuli with two levels of PE and difficulty were presented on a gray background. Here, only PE and noise

\section{Experiment 1A}

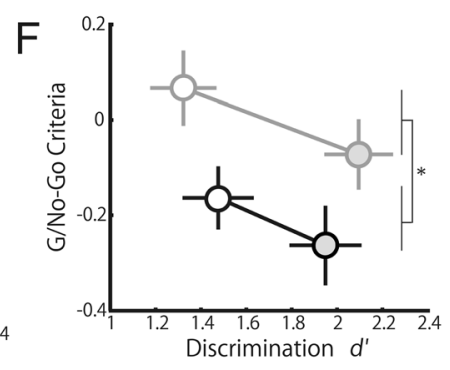

Moreover, these patients claim that they are just guessing that is, performing visual tasks with no subjective confidence. Incidentally, when perceptual capacity $\left(d^{\prime}\right)$ is controlled for, both subjective confidence and awareness seem to depend on prefrontal activity (Fleming \& Dolan, 2012; Lau \& Passingham, 2006). Because many dominant theories (Dehaene \& Naccache, 2001; Jack \& Shallice, 2001; Norman \& Shallice, 1986) have suggested that prefrontal cortex and conscious awareness may play a critical role in cognitive control functions and, more directly, because perceptual confidence also relies on areas of prefrontal cortex (Fleming \& Dolan, 2012; Lau \& Passingham, 2006) involved in cognitive control, here we tested whether perceptual confidence affects two representative cognitive functions: response inhibition performance (Exps. 1A and 1B) and task set preparation (Exps. 2A and 2B). Surprisingly, we will show that higher perceptual confidence does not enhance overall cognitive control performance. That is, stimuli perceived with high and low confidence triggered the participants' capacities for inhibitory control and task set preparation to similar degrees.

\section{Method}

Participants Twenty-four students (17 female, seven male; mean age 2.7 years) from Columbia University participated.

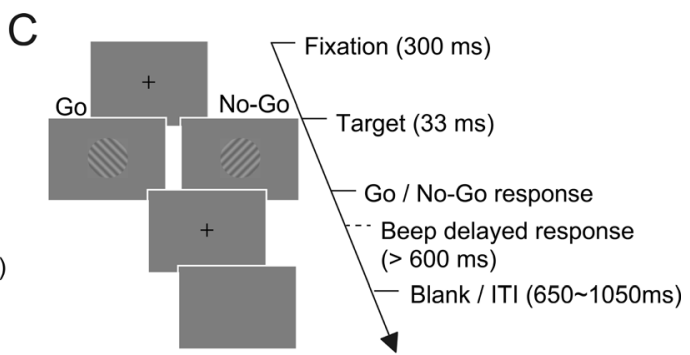

are shown, for demonstrative purposes. The stimuli served as either a go or a no-go signal, depending on their tilts. The trial sequence was divided into durations shown in parentheses. (D) Discrimination $d^{\prime}$ and confidence ratings in Experiment 1A. (E, F) Mean go/no-go $d^{\prime}$ and go/no-go criteria as functions of $\mathrm{PE}$ and difficulty levels, plotted against discrimination $d^{\prime}$. Error bars indicate standard errors of the means. Asterisks $\left(^{*} p<\right.$ $\left..05,{ }^{* *} p<.01\right)$ are shown only for comparisons between high and low PE levels (see the Results for the effects of difficulty level). 
A

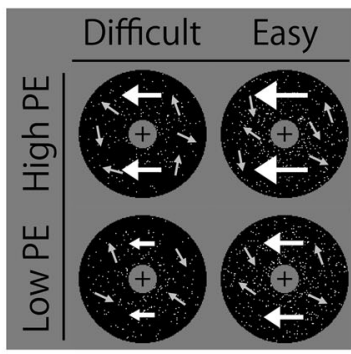

B

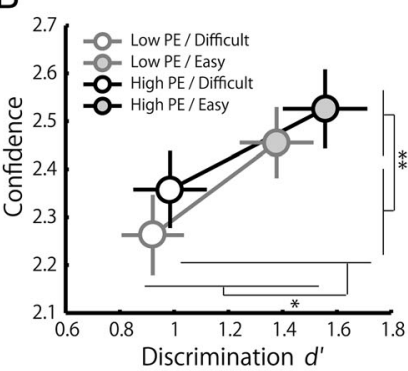

D
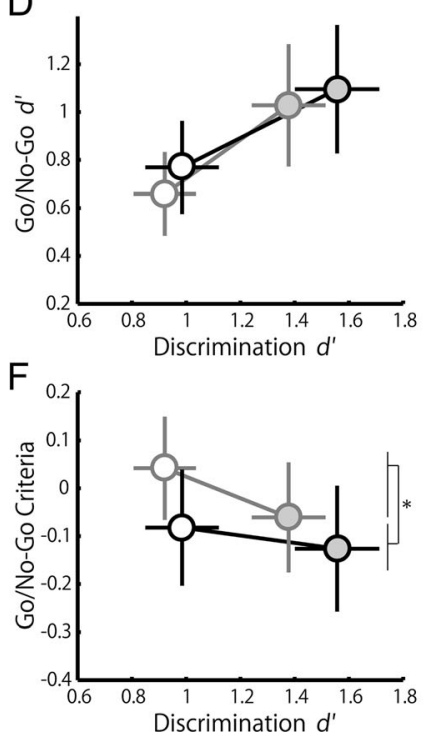

C

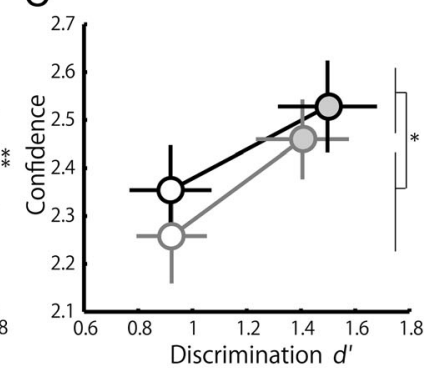

E
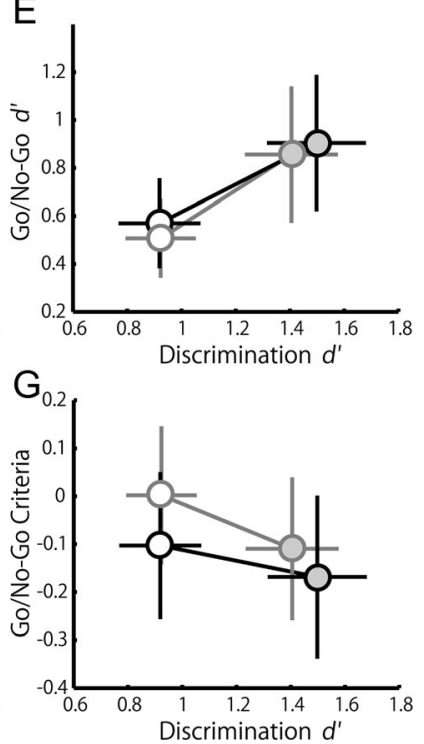

Fig. 2 (A) Dot motion stimuli from Experiments 1B and 2B. Each stimulus consisted of PE (the proportion of dots moving toward the left or right) and noise (the proportion of dots moving in random directions). Stimuli had either low or high levels of PE, with two difficulty levels. White large arrows schematically illustrate the numbers of dots for PE, and gray small arrows illustrate the numbers of dots for noise. The total number of dots varied across the stimulus types. (B-G) Results from Experiment 1B: Discrimination confidence is plotted against $d^{\prime}$ for all 19 participants (B) and for the selected 14 participants (C). Go/no-go $d^{\prime}$ is plotted against discrimination $d^{\prime}$ for all participants (D) and for the selected participants (E). Go/no-go criteria for all participants (F) and for the selected participants (G) are also plotted against discrtimination $d^{\prime}$. Error bars indicate standard errors of the means. Asterisks $\left({ }^{*} p<.05\right.$, $\left.{ }^{* *} p<.01\right)$ are shown only for comparisons between high and low PE levels (see the Results for the effects of difficulty level).

They received $\$ 10 / \mathrm{h}$ and completed a written informed consent form prior to participation. The study was approved by the Columbia University's Committee for the Protection of Human Subjects.

Stimulus The stimulus was a set of superimposed left- and right-tilted gratings embedded in noise. Participants reported the tilt of the strongest grating (Fig. 1A). Here we define positive evidence $(\mathrm{PE})$ as the evidence favoring a correct stimulus identification response, and negative evidence (NE) as the evidence favoring the incorrect response. For instance, in the grating stimuli, PE would be sensory tilt evidence driven mainly by the higher-contrast grating, which would support the correct tilt response, whereas NE would be the tilt evidence driven mainly by the lower-contrast grating, which would support the incorrect tilt response. Note that this stimulus-based definition of PE and NE is different from previous, response-based definitions of PE and NE (Maniscalco \& Lau, 2012; Zylberberg et al., 2012). In the context of using ambiguous stimuli as go/no-go signals, it would be difficult to discern whether a "go" response (e.g., to a left-tilting grating) was generated by a true perceptual decision that the grating was tilted left or by a failure to inhibit the "left" response (i.e., the "go" response), even though the true perceptual decision was that the grating was tilting right. Thus, response-based notions of PE and NE might encounter difficulties in the present go/no-go paradigms, and we use the stimulus-based definitions for clarity of exposition.

The stimulus was a circle $\left(3^{\circ}\right.$ diameter) containing a target grating (PE), a nontarget grating (NE), and random dot noise. $\mathrm{PE}$ was a sinusoidal grating ( 2 cycles/deg) tilted left or right at a 45-deg angle. NE was another sinusoidal grating with an orientation orthogonal to that of PE. The stimuli had either a high or a low level of PE (i.e., high or low contrast for the target grating), which was intended to yield relatively high and low confidence levels, respectively. Within each PE level, we further created trials that had high or low ratios of PE contrast to NE contrast, so as to yield relatively high and low perceptual accuracy (i.e., easy and difficult). Hence, a given stimulus had one of the four combinations of difficulty (easy/difficult) and PE level (high/low). The Michelson (1927) contrast levels (i.e., the difference between the maximum and minimum luminosity, divided by the average between the two luminosities) for $\mathrm{PE}\left(\mathrm{C}_{\mathrm{PE}}\right), \mathrm{NE}\left(\mathrm{C}_{\mathrm{NE}}\right)$, and noise $\left(\mathrm{C}_{\text {noise }}\right)$ were calibrated for each participant. The stimuli were generated and presented on a gray background with the Psychophysics Toolbox (Brainard, 1997) in MATLAB.

Calibration block After two practice blocks (28 trials each), participants underwent a calibration block (80 trials). They were instructed to discriminate grating tilt and then to rate their confidence in the discrimination response using a fourpoint scale (Fig. 1B). With the grating stimuli, participants were instructed to indicate the tilt of higher contrast. Responses were entered on a computer keyboard.

On each trial, a black crosshair $\left(0.35^{\circ}\right.$ wide $)$ was presented at the center of the screen on a gray background for $300 \mathrm{~ms}$. The crosshair was replaced by a grating stimulus $(33 \mathrm{~ms})$, 
which was followed by another crosshair. Participants discriminated the grating tilt by pressing the "1" or " 2 " key to indicate a left or a right orientation, respectively. After the discrimination response, the black crosshair turned gray. Participants then rated their confidence in their orientation judgments on a four-point scale, by pressing one of four horizontally aligned keys ("7"/“8"/“9"/“0" on the keyboard). After the confidence rating was entered, the crosshair disappeared from the screen and the next trial began after $750 \mathrm{~ms}$. Participants had a 5-s time limit after stimulus offset to enter their discrimination response, and a separate 5-s time limit after the entry of the discrimination response to enter the confidence rating. Trials in which either of the time limits expired were excluded from the analysis ( $0.5 \%$ of the overall trials). Participants were instructed to stay fixated on the crosshair throughout the trial. In the discrimination block, the trial structure was identical to that in the calibration block.

During the calibration block, the ratio of $\mathrm{C}_{\mathrm{PE}}$ relative to $\mathrm{C}_{\text {noise }}\left(\mathrm{C}_{\mathrm{PE}} / \mathrm{C}_{\text {noise }}\right.$ ratio $)$ in the grating stimuli was continuously adjusted to achieve a discrimination accuracy of $84 \%$ with the QUEST threshold estimation procedure (Watson \& Pelli, 1983). The grating stimuli were composed of PE, NE, and noise components, each with its own level of Michelson contrast $\mathrm{C}$.

We defined

$\mathrm{C}_{\text {total }}=\mathrm{C}_{\mathrm{PE}}+\mathrm{C}_{\mathrm{NE}}+\mathrm{C}_{\text {noise }}$,

$\mathrm{R}_{\mathrm{PE} / \text { noise }}=\mathrm{C}_{\mathrm{PE}} / \mathrm{C}_{\text {noise }}$,

$\mathrm{R}_{\mathrm{NE} / \mathrm{PE}}=\mathrm{C}_{\mathrm{NE}} / \mathrm{C}_{\mathrm{PE}}$.

Then, the contrast for each component could be expressed as

$\mathrm{C}_{\text {noise }}=\quad \mathrm{C}_{\text {total }} / \quad\left(\mathrm{R}_{\mathrm{PE} / \text { noise }}+\mathrm{R}_{\mathrm{PE} / \text { noise }} \times \mathrm{R}_{\mathrm{NE} / \mathrm{PE}}+1\right) \quad$,

$\mathrm{C}_{\mathrm{PE}}=\left(\mathrm{R}_{\mathrm{PE} / \text { noise }} \times \mathrm{C}_{\text {total }}\right) /\left(\mathrm{R}_{\mathrm{PE} / \text { noise }}+\mathrm{R}_{\mathrm{PE} / \text { noise }} \times \mathrm{R}_{\mathrm{NE} / \mathrm{PE}}+1\right)$,

$\mathrm{C}_{\mathrm{NE}}=\left(\mathrm{R}_{\mathrm{NE} / \mathrm{PE}} \times \mathrm{R}_{\mathrm{PE} / \text { noise }} \times \mathrm{C}_{\text {total }}\right) /\left(\mathrm{R}_{\mathrm{PE} / \text { noise }}+\mathrm{R}_{\mathrm{PE} / \text { noise }} \times \mathrm{R}_{\mathrm{NE} / \mathrm{PE}}+1\right)$.

$\mathrm{C}_{\text {total }}$ was set to 1 . Thus, the contrasts of the PE, NE, and noise components of the stimulus could be uniquely determined by setting values for the two parameters $\mathrm{R}_{\mathrm{PE} / \text { noise }}$ and $\mathrm{R}_{\mathrm{NE} / \mathrm{PE}}$.

The calibration block contained two randomly interleaved sets of 40 trials, in which each set had a high (.7) or a low (.35) value for $\mathrm{R}_{\mathrm{NE} / \mathrm{PE}}$. During the calibration block, $\mathrm{R}_{\mathrm{PE} / \text { noise }}$ was adjusted continuously with the QUEST threshold estimation procedure (Watson \& Pelli, 1983) in order to determine the value that would yield $84 \%$ correct responses in the tilt discrimination task. Separate values for $\mathrm{R}_{\mathrm{PE} / \text { noise }}$ were estimated for the two different levels of $\mathrm{R}_{\mathrm{NE} / \mathrm{PE}}$.

For trials with the higher value of $\mathrm{R}_{\mathrm{NE} / \mathrm{PE}}$, the distractor $\mathrm{NE}$ component was more salient than the PE component, which in turn necessitated a higher value for $\mathrm{R}_{\mathrm{PE} / \text { noise }}$ (i.e., a $\mathrm{PE}$ component that was more salient than the noise) in order to achieve tilt discrimination accuracy of $84 \%$ correct. Thus, the QUEST procedure for trials with $\mathrm{R}_{\mathrm{NE} / \mathrm{PE}}=.7$ yielded a higher value of $\mathrm{R}_{\mathrm{PE} / \text { noise }}$ (call it $\mathrm{R}_{\mathrm{High}-\mathrm{PE} / \text { noise), and thus a }}$ higher value of $\mathrm{C}_{\mathrm{PE}}$ (call it $\mathrm{C}_{\mathrm{High}-\mathrm{PE}}$ ). Conversely, the QUEST procedure for trials with $\mathrm{R}_{\mathrm{NE} / \mathrm{PE}}=.35$ yielded a lower

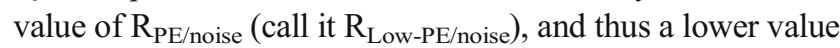
of $\mathrm{C}_{\mathrm{PE}}$ (call it $\mathrm{C}_{\text {Low-PE}}$ ). Nonetheless, both stimulus sets were calibrated to have the same level of discrimination performance of $\sim 84 \%$ correct. In this way, we created stimuli with different PE levels but similar task performance.

Task difficulty was modulated by multiplying the original values of $\mathrm{R}_{\mathrm{PE} / \text { noise }}$ achieved via calibration by either 1 or .7 . The latter case leads to a less salient PE component, and thus inferior tilt discrimination performance (i.e., difficult condition).

In summary, we created stimulus sets that varied across the factors PE Level (high/low) and Difficulty (easy/difficult), with the intention of achieving similar levels of tilt discrimination performance across PE levels for each difficulty level. The stimulus sets were characterized by the following settings:

Low PE, easy : $\mathrm{R}_{\mathrm{NE} / \mathrm{PE}}=.35, \mathrm{R}_{\mathrm{PE} / \text { noise }}=\mathrm{R}_{\text {Low-PE/noise }}^{*}$, Low PE, difficult: $\mathrm{R}_{\mathrm{NE} / \mathrm{PE}}=.35, \mathrm{R}_{\mathrm{PE} / \text { noise }}=.7 \times \mathrm{R}^{*}{ }_{\text {Low-PE } / \text { noise }}$, High P E, easy : $\mathrm{R}_{\mathrm{NE} / \mathrm{PE}}=.7, \mathrm{R}_{\mathrm{PE} / \text { noise }}=\mathrm{R}^{*}{ }_{\text {High-PE } / \text { noise }}$, High PE, difficult : $\mathrm{R}_{\mathrm{NE} / \mathrm{PE}}=.7, \mathrm{R}_{\mathrm{PE} / \text { noise }}=.7 \times \mathrm{R}^{*}{ }_{\text {High-PE } / \text { noise }}$,

where $\mathrm{R}_{\text {Low-PE/noise }}$ and $\mathrm{R}_{\text {High-PE/noise }}$ refer to the values for $\mathrm{R}_{\mathrm{PE} / \text { noise }}$ estimated via QUEST in the calibration block to achieve $\sim 84 \%$ correct performance for $\mathrm{R}_{\mathrm{NE} / \mathrm{PE}}=.35$ and $\mathrm{R}_{\mathrm{NE} / \mathrm{PE}}=.7$, respectively.

Discrimination blocks Following the calibration block, participants underwent two discrimination blocks, one before and one after the go/no-go block (see below). The task was the same as in the calibration block. In each block, stimuli with the four combinations of PE and difficulty levels $(2 \mathrm{PE} \times 2$ Difficulty) were randomly distributed across 480 trials. Gratings with calibrated contrast levels served as the stimuli. The trial structures were identical to the ones used in the calibration blocks. Participants took a brief break $(<1 \mathrm{~min})$ after every 100 trials. The mean $d$ 's did not differ between the first $(M=1.77 \pm .58)$ and second $(M=1.64 \pm .71)$ discrimination blocks $[t(23)=1.29, p=.21]$, suggesting that no fatigue or practice effects influenced the data across blocks. Hence, the performance from the two blocks was collapsed in the subsequent analyses.

Go/no-go blocks In the go/no-go task, the calibrated stimuli served as go and no-go signals, depending on the orientations of grating tilt: One of the target stimulus (PE) orientations (left or right, counterbalanced across participants) served as a go signal, and the other served as a no-go signal. The numbers of trials with go and no-go signals were matched (200 each). Note that whereas relatively infrequent go trials prompt a 
tendency to make "go" responses (Chikazoe et al., 2009), previous studies have also used matched numbers of trials (van Gaal, Ridderinkhof, Scholte, \& Lamme, 2010). We particularly matched the trial numbers in order to enhance the statistical power for both go and no-go trials. On each trial (Fig. 1C), a black fixation crosshair (300 ms) was followed by the go/no-go signal. The go/no-go signal (grating) was presented for $33 \mathrm{~ms}$, and the signal was then replaced by a black crosshair. Upon go signal presentation, participants were required to press a key as quickly as possible. With no-go signals, participants were required to withhold key pressing. Regardless of the signal type, auditory feedback was given when participants made a slow response, occurring more than $600 \mathrm{~ms}$ from the signal offset for those trials in which a "go" response was entered. When a key was pressed or when the response window of $600 \mathrm{~ms}$ lapsed, the crosshair disappeared from the screen, and the next trial began after an interval that was randomly jittered from 650 to $1,050 \mathrm{~ms}$.

Analyses Discrimination $d^{\prime}$ was calculated for each of the four grating types ( $2 \mathrm{PE} \times 2$ difficulty levels) with standard signal detection theory (SDT) methods (Macmillan \& Creelman, 2005). The mean confidence rating was calculated for each stimulus type. The means for discrimination $d^{\prime}$ and confidence rating were calculated separately for Discrimination Blocks 1 and 2. The means across the blocks were used for further analyses for each experiment. The means for discrimination $d^{\prime}$ and confidence (Fig. 1D) were independently analyzed in a $2(\mathrm{PE}$ level $) \times 2$ (difficulty level) repeated measures analysis of variance (ANOVA).

Go/no-go performance was also calculated with SDT (Macmillan \& Creelman, 2005): The hit rate (i.e., percentage of correct "go" responses, on go trials) and false alarm rate (i.e., percentage of incorrect "go" responses, on no-go trials) were calculated separately for the four stimulus types $(2 \mathrm{PE} \times$ 2 difficulty levels). From the hit rate and false alarm rate, the go/no-go $d^{\prime}$ (Fig. 1E) and criterion (Fig. 1F) were calculated with SDT (MacMillan \& Creelman, 2005) as

$d^{\prime}=z$ (hit rate) $-z$ (false alarm rate) ,

criteria $=-.5 \times[z$ (hit rate $)+z($ false alarm rate $)]$.

Here, $z$ denotes the inverse of the normal cumulative distribution function. Go/no-go $d^{\prime}$ and criterion were analyzed independently in a $2(\mathrm{PE}$ level) $\times 2$ (difficulty level) repeated measures ANOVA. Furthermore, the mean reaction time (RT, from the go/no-go signal offset) for the "go" responses were calculated for each stimulus type ( $2 \mathrm{PE} \times 2$ difficulty levels) and analyzed in another independent ANOVA.

Results

Discrimination blocks For confidence rating, we found a significant main effect of PE level [ANOVA: $F(1,23)=$
7.81, $\left.p<.01, \eta_{\mathrm{p}}{ }^{2}=.25\right]$, revealing that the stimuli with high PE yielded higher confidence ratings $(M=2.42 \pm$ $0.54)$ than did the stimuli with low PE $(M=2.17 \pm$ $0.50)$. A main effect of difficulty level was also significant $\left[F(1,23)=35.00, p<.001, \eta_{\mathrm{p}}{ }^{2}=.60\right]$, showing that confidence was higher in the easy than in the difficult condition, as expected. The interaction was not significant $\left[F(1,23)=2.36, p=.14, \eta_{\mathrm{p}}{ }^{2}=.09\right]$, suggesting that higher $\mathrm{PE}$ led to higher confidence regardless of difficulty levels. Thus, we successfully manipulated confidence independently from $d^{\prime}$ (Fig. 1D).

As for the discrimination $d^{\prime}$, easy conditions led to higher $d$ 's than did difficult conditions, as expected $\left[F(1,23)=54.75, p<.001, \eta_{\mathrm{p}}{ }^{2}=.70\right]$. A significant interaction emerged between $\mathrm{PE}$ and difficulty levels $\left[F(1,23)=5.98, p=.02, \eta_{\mathrm{p}}{ }^{2}=.21\right]$. The interaction arose from the fact that the difference in $d$ 's between the two difficulty levels was significantly larger for low $\mathrm{PE}(M=.77)$ than for high $\mathrm{PE}(M=.47)[t(23)=2.45$, $p=.02]$ (Fig. 1D). The main effect of PE was not significant $(p=.98)$.

Go/no-go blocks Our analysis of go/no-go $d$ ' revealed that the main effect of PE was not significant (Fig. 1E) $(p>.10)$. That is, whereas the higher PE level led to higher confidence ratings with matched $d^{\prime}$ in the discrimination task, it did not lead to a higher go/no-go $d^{\prime}$. As expected, the main effect of difficulty level was significant $\left[F(1,23)=48.46, p<.001, \eta_{\mathrm{p}}{ }^{2}=\right.$ .68]. The interaction for go/no-go $d^{\prime}$ was not significant $(p=$ .13). Although there was no effect of PE level on go/no-go $d^{\prime}$, the analysis of go/no-go criterion revealed a significant main effect of $\operatorname{PE}\left[F(1,23)=5.40, p=.03, \eta_{\mathrm{p}}{ }^{2}=.19\right]$, showing that higher PE led to a lower (more liberal) response criterion (Fig. 1F). Easy conditions, which led to higher confidence and discrimination $d^{\prime}$ than did difficult conditions, led to a lower response criterion $\left[F(1,23)=13.44, \eta_{\mathrm{p}}{ }^{2}=.37\right]$. The interaction was not significant $(p=.48)$.

The mean RTs to make "go" responses were as follow: high-PE/difficult $(M=446 \mathrm{~ms} \pm 11)$, high-PE/easy $(M=$ $438 \mathrm{~ms} \pm 10)$, low-PE/difficult $(M=462 \mathrm{~ms} \pm 13)$, low-PE/ easy $(M=446 \mathrm{~ms} \pm 12)$. There was no significant main effect of PE level $\left[F(1,23)=2.48, p=.13, \eta_{\mathrm{p}}{ }^{2}=.10\right]$. The main effect of difficulty level was significant $[F(1,23)=5.24, p=$ $\left..03, \eta_{\mathrm{p}}{ }^{2}=.19\right]$, due to shorter RTs on easy than on difficult conditions. The interaction between PE level and difficulty level was not significant $[F(1,23)=0.11, p$ $\left.=.31, \eta_{\mathrm{p}}{ }^{2}=.04\right]$. The results were similar when only the trials with hits (i.e., "go" responses on the go trials) or false alarms (i.e., "go" responses on no-go trials) were considered. Overall, these results suggest that although accuracy in perceiving the cues $\left(d^{\prime}\right)$ facilitated the speed to perform the go/no-go task, perceptual confidence led to no noticeable effect. 


\section{Experiment 1B}

Experiment 1A showed that perceptual confidence has a minimal effect on go/no-go task performance. We conducted Experiment 1B to reexamine the effect of perceptual confidence with a different stimulus set (dot motion).

\section{Method}

Participants Nineteen students (nine female, 10 male; mean age 24.9 years) from Columbia University participated in the same manner as in Experiment 1A.

Stimuli The stimulus (Fig. 2A) was a set of white dots moving at the speed of $6 \mathrm{deg} / \mathrm{s}$ within a dark gray annulus (outer circle diameter $=3^{\circ}$, inner circle radius $=.35^{\circ}$ ). Each stimulus was presented for $66 \mathrm{~ms}$ (four frames at $60 \mathrm{~Hz}$ ). The dots had infinite lifetimes, and those that moved outside the annulus were replaced at a random location within the annulus. A crosshair $\left(0.35^{\circ}\right.$ wide $)$ was presented within the inner circle throughout each trial. $P E$ was defined as the number of dots that coherently moved left or right, and noise constituted the remaining dots, which moved in random directions. The numbers of dots serving as PE and noise were determined for each participant in a calibration block. Note that whereas NE was a grating whose orientation was opposite that of $\mathrm{PE}$ in Experiment $1 \mathrm{~A}$, here NE was a set of randomly moving dots (i.e., noise). We introduced this new NE manipulation to further strengthen our results: If we always used an NE (e.g., leftoriented) that conflicted with PE (e.g., right-oriented), as in Experiment 1A, our results could be challenged on the ground that stimulus conflict confounded perceptual confidence. Yet, if we could replicate the results using another method to manipulate NE (i.e., via random noise), we could argue against stimulus conflict confounding the results. The stimuli were generated and presented on a gray background with the Psychophysics Toolbox (Brainard, 1997) in MATLAB. Since performance $\left(d^{\prime}\right)$ did not differ statistically between the first $(M=1.31 \pm 0.53)$ and second $(M=1.11 \pm 0.74)$ discrimination blocks $[t(18)=1.43, p=.17]$, suggesting no fatigue or practice effects on the data across blocks, the data from the two blocks were collapsed in the subsequent analyses.

Calibration block Following two practice blocks (28 trials each), participants underwent a calibration block (80 trials) with the dot motion stimuli. In the dot motion stimuli, the ratio of the PE dot number to the noise dot number was continuously adjusted to achieve a discrimination accuracy of $84 \%$ with the QUEST threshold estimation procedure (Watson \& Pelli, 1983). The density of dots was fixed at $70 \mathrm{dots} / \mathrm{deg}^{2}$ during the calibration block. Two sets of 40 trials were interleaved within the block to independently yield two estimates of the PE/noise ratio. Participants were instructed to indicate the direction toward which the majority of dots were moving.

These estimates were used to determine the numbers of dots serving as PE and noise in the subsequent tasks. There were four types of stimuli, which had one of the two PE levels (high/low) and two difficulty levels (easy/difficult). For highPE/easy stimuli, the mean of the two PE/noise ratio estimates was used to determine the numbers of dots serving as $\mathrm{PE}$ and noise with the density of $70 \mathrm{dots} / \mathrm{deg}^{2}$. For low-PE/easy stimuli, the numbers of PE dots and noise dots used in the high-PE/ easy stimuli were each multiplied by .5 to determine the PE dot and noise dot numbers, respectively. For both the high-PE and low-PE difficult stimuli, the numbers of PE dots were determined on the basis of the PE dot number for easy stimuli with the same PE level (high/low): For each PE level, the number of PE dots in easy stimuli was multiplied by .5 for six participants and by .6 for 13 participants, to reduce the number of dots serving as PE and make the stimulus discrimination more difficult. As a result, the densities of dots varied across the four types of stimuli ( $2 \mathrm{PE} \times 2$ difficulty), depending on the numbers of dots for $\mathrm{PE}$ and noise.

Discrimination and go/no-go blocks The discrimination blocks were identical to those of Experiment 1A, except that the task was to indicate the direction toward which the majority of dots were moving. In the go/no-go block, the difference from Experiment $1 \mathrm{~A}$ was that the directions of dot motion, left or right, served as the go/no-go signal. In both the discrimination and go/no-go blocks, the dot motion stimuli were presented for 66 instead of $33 \mathrm{~ms}$, to ensure motion direction perception.

Analyses In all experimental blocks, the data were processed as in Experiment 1A, with one exception: Data from the go/ no-go task were analyzed from 14 participants, selected on the basis of their discrimination task performance in order to selectively examine the effects of perceptual confidence (see below).

\section{Results}

Discrimination blocks The overall results replicated those of Experiment 1A. For confidence ratings, we observed significant main effects of difficulty and PE level $[F(1,18)=21.59$, $p<.001, \eta_{\mathrm{p}}{ }^{2}=.55 ; F(1,18)=7.69, p=.013, \eta_{\mathrm{p}}{ }^{2}=.30$, respectively], revealing higher confidence ratings with lower difficulty level as well as for higher PE level (Fig. 2B). The interaction was not significant $\left[F<1, p>.10, \eta_{\mathrm{p}}{ }^{2}=.03\right]$. For discrimination $d^{\prime}$, a significant main effect of difficulty was apparent $\left[F(1,18)=84.43, p<.001, \eta^{2}=.82\right]$, as well as an effect of PE $\left[F(1,18)=7.46, p=.014, \eta_{\mathrm{p}}^{2}=.29\right]$, indicating higher $d^{\prime}$ with lower difficulty level and higher PE level. The interaction was not significant $\left[F=2.4, p=.14, \eta_{\mathrm{p}}{ }^{2}=.12\right]$. 
These results show that while increasing the PE level did successfully increase confidence, it also increased $d^{\prime}$. Thus, to tease apart the effects of confidence and $d^{\prime}$ on go/no-go task performance, we eliminated the five participants who had the largest $d^{\prime}$ difference between the high and low PE levels (i.e., one standard error above the mean $d^{\prime}$ difference across all participants). With the remaining 14 participants (Fig. 2C), the $d^{\prime}$ difference between high and low PE levels was no longer significant $(p=.21)$, whereas the $d^{\prime}$ difference between the easy and difficulty conditions remained significant, as expected $\left[F(1,13)=51.49, p<.001, \eta_{\mathrm{p}}{ }^{2}=.80\right]$. With this subset of participants, confidence ratings were still higher for a high $(M=2.44 \pm 0.34)$ than for a low $(M=2.40 \pm 0.33)$ PE level $\left[F(1,13)=4.84, p=.046, \eta_{\mathrm{p}}{ }^{2}=.27\right]$, as well as for easy than for difficult conditions $\left[F(1,13)=14.82, p=.002, \eta_{\mathrm{p}}{ }^{2}=.53\right]$. Note that it would be expected to find a smaller confidence difference between the PE levels after eliminating the five participants, given that the eliminated participants had higher confidence at the high PE level, due to an unintended increase in $d^{\prime}$ in addition to higher PE level. Since the selected participants had higher confidence with higher PE level, despite matched $d$ 's, their data were submitted to the subsequent analyses of go/no-go task performance to examine the effects of perceptual confidence.

Go/no-go blocks Replicating Experiment 1A, the analysis of go/no-go $d^{\prime}$ (Fig. 2E) from the selected participants revealed a main effect of difficulty level $\left[F(1,13)=7.87, p=.015, \eta_{\mathrm{p}}{ }^{2}=\right.$ $.38]$, indicating higher go/no-go $d^{\prime}$ for the easy conditions. The main effect of PE level and the interaction between PE and difficulty levels were both not significant $(p s>.10)$. For the go/no-go criterion (Fig. $2 \mathrm{G}$ ), the main effect of difficulty level was significant $\left[F(1,13)=9.81, p<.01, \eta_{\mathrm{p}}{ }^{2}=.43\right]$, indicating a more liberal response criterion in easy than in difficult conditions. The main effect of PE level and the interaction were not significant $(p>.10)$.

When the data from all 19 participants (i.e., including the five participants excluded for having a large $d^{\prime}$ difference between PE conditions) were analyzed, the results for go/no-go $d^{\prime}$ remained qualitatively similar (Fig. 2D). The results for the go/no-go criterion differed in that the main effect of PE level became significant $\left[F(1,18)=5.25, p=.034, \eta_{\mathrm{p}}{ }^{2}=.23\right]$, indicating a more liberal response criterion with higher PE level (Fig. 2F). Considering that higher PE level led to higher confidence ratings, this result is in agreement with the results from Experiment 1A, in which a higher PE level (also associated with higher confidence) led to more liberal criteria.

The mean RTs among the selected 14 participants were as follow: high-PE/difficult $(M=387 \mathrm{~ms} \pm 45)$, high-PE/easy ( $M$ $=393 \mathrm{~ms} \pm 46)$, low-PE/difficult $(M=394 \mathrm{~ms} \pm 41)$, and lowPE/easy $(M=392 \mathrm{~ms} \pm 33)$. The analyses of mean RTs revealed no effect of PE level $\left[F(1,13)=0.29, p=.60, \eta_{\mathrm{p}}{ }^{2}=\right.$ $.02]$. A main effect of difficulty level $[F(1,13)=0.21, p=.66$, $\left.\eta_{\mathrm{p}}{ }^{2}=.02\right]$ and the interaction between PE level and difficulty level $\left[F(1,13)=0.35, p=.56, \eta_{\mathrm{p}}{ }^{2}=.03\right]$ were also not significant. Although the same analysis in Experiment 1A had revealed a significant main effect of difficulty level, such an effect was not observed in this experiment. This was likely due to the fact that, whereas a static stimulus (i.e., grating) was used in Experiment 1A, a dynamic stimulus (i.e., motion) was used in this experiment. Since the dynamic stimulus had a less clear onset of the cue (i.e., the time to perceive the motion direction varied across trials), the effect of discrimination accuracy on "go" response RTs might have diffused. The results remained the same when all 19 of the participants were considered, as well as when only the trials with hits (i.e., "go" responses on go trials) or false alarms (i.e., "go" responses on no-go trials) were considered. Overall, these results suggest that confidence in perceiving the cues $\left(d^{\prime}\right)$ does not increase the speed to perform a go/no-go task.

\section{Experiment 2A}

Experiments $1 \mathrm{~A}$ and $1 \mathrm{~B}$ showed no notable effects of perceptual confidence on cognitive control capacity using a go/no-go paradigm. We conducted Experiments $2 \mathrm{~A}$ and $2 \mathrm{~B}$ to further examine the effect of perceptual confidence on another cognitive control function-namely, task set preparation. Experiments 2A and 2B were similar except that they used the grating stimuli and dot motion stimuli, respectively.

\section{Method}

Participants Nineteen students (10 female, nine male; mean age 21.7 years) from Columbia University participated in the same manner as in Experiments 1A and 1B.

Calibration and discrimination blocks Participants underwent the calibration and discrimination blocks in the same manner as in Experiment 1A, with one exception: A black letter $\mathrm{X}\left(0.27^{\circ}\right.$ wide $)$ followed the grating stimulus with a stimulus onset asynchrony (SOA) of $600 \mathrm{~ms}$. The letter was inserted so as to calibrate the grating contrast and to measure discrimination accuracy and confidence in a trial sequence similar to the one used in the task set blocks (see below). Upon presentation of the letter, participants made a forced discrimination of the tilt of the higher-contrast grating by pressing the " 1 " or the " 2 " key to indicate a left or right tilt, respectively. After the response, the letter was replaced by a crosshair. Since performance $\left(d^{\prime}\right)$ did not differ statistically between the first $(M=1.92 \pm$ $0.44)$ and second $(M=1.69 \pm 0.65)$ discrimination blocks $[t(18)=1.54, p=.13]$, performance was collapsed across the blocks in the subsequent analyses. 
Task set block The grating tilts (left/right, counterbalanced) cued which of the two tasks (phonological/numerical) was to be performed on the trials. In the phonological task, participants were asked to judge whether the presented letter (five vowels $/ \mathrm{h} / \mathrm{m} / \mathrm{k} / \mathrm{p} / \mathrm{y}$ ) was a vowel or a consonant (Fig. 3A). In the numerical task, they were asked to judge whether the presented number (1-10) was odd or even. They were instructed to use the stimulus tilts as task cues to perform the tasks as quickly and accurately as possible. On each trial, one of the target letters or numbers $\left(0.27^{\circ}\right.$ wide) followed the presentation of a cue grating, with three possible SOAs (400/600/ $800 \mathrm{~ms}$ ). Auditory feedback followed incorrect or omitted responses within a 4-s window. The task set block consisted of a total of 540 trials, 480 of which had stimuli with one of the four combinations of PE and difficulty levels. The remaining 60 trials were dummy trials with a highly visible PE (40\% contrast without NE, yielding near $100 \%$ discrimination accuracy), to remind participants of the assigned associations between grating tilt and task type.

\section{Results}

Discrimination blocks Discrimination $d^{\prime}$ was calculated and analyzed as in Experiments 1A and 1B.

For the confidence ratings, a 2 (PE level) $\times 2$ (difficulty level) repeated measures ANOVA revealed a significant main effect of PE level $\left[F(1,18)=26.09, p<.001, \eta_{\mathrm{p}}{ }^{2}=.59\right]$, showing that confidence ratings became higher with higher PE level. The main effect of difficulty was also significant $\left[F(1,18)=30.20, p<.001, \eta_{\mathrm{p}}{ }^{2}=.63\right]$, showing that confidence was higher for easy than for difficult conditions. The interaction was not significant $\left[F(1,18)=2.95, p=.10, \eta_{\mathrm{p}}{ }^{2}=\right.$ $.14]$.

As for discrimination $d^{\prime}$, a 2 (PE level) $\times 2$ (difficulty level) ANOVA revealed no significant main effect of PE level on discrimination $d^{\prime}\left[F(1,18)=2.92, p=.11, \eta_{\mathrm{p}}{ }^{2}=.14\right]$, as is shown in Fig. 3B. Yet, it did reveal a significant interaction between PE level and difficulty level $[F(1,18)=52.36, p<$ $\left..01, \eta_{\mathrm{p}}{ }^{2}=.74\right]$ : Whereas discrimination $d^{\prime}$, along with confidence, was elevated with higher PE level for difficult stimuli (Bonferroni: $p<.001$ ), discrimination $d^{\prime}$ did not differ between the two PE levels for easy stimuli (Bonferroni: $p=$ .12). Thus, the elevation of confidence for high-PE stimuli did not covary with discrimination $d^{\prime}$ for easy stimuli, but it did for difficult stimuli. As expected, there were main effects of difficulty/easy stimuli $\left[F(1,18)=52.39, p<.001, \eta_{\mathrm{p}}{ }^{2}=\right.$ $.74]$, showing that the easy condition led to higher $d^{\prime}$ than the difficult condition.

Task set block Means for the cued task RTs (Fig. 3C) were calculated for correct trials whose RTs were within three standard deviations of the mean $(3.8 \%$ of trials were eliminated for incorrectness, and an additional $2.1 \%$ were eliminated for
A

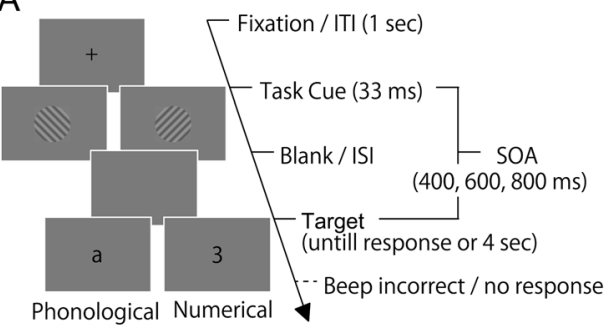

B

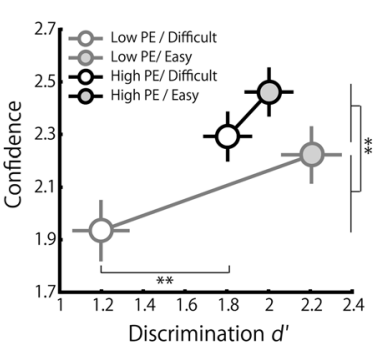

C
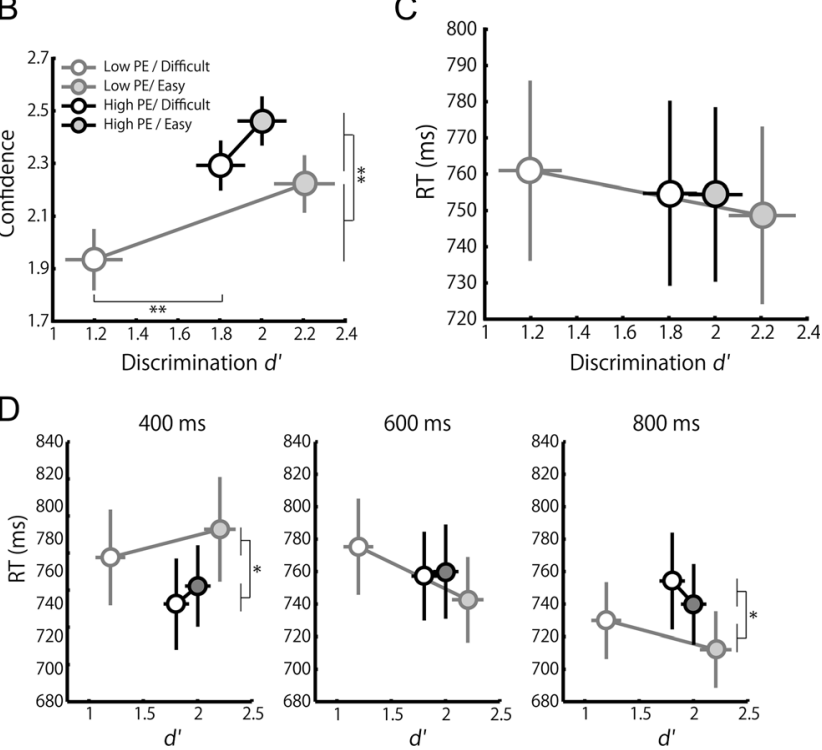

Fig. 3 (A) Task-cueing paradigm in Experiment 2A. Stimuli with various levels of PE and difficulty were presented on a gray background. Here, only PE and noise are shown, for demonstrative purposes. The tilt of the stimuli forecasted the following task type, which was either a phonological or numerical judgment. The trial sequence was divided into the durations shown in parentheses. (B) Discrimination $d^{\prime}$ and confidence ratings in Experiment 2A. (C-D) Results from the task set block: (C) Mean RTs (in milliseconds) of the cued task as a function of PE and NE levels, plotted against discrimination $d^{\prime}$. (D) Mean cued-task RTs across stimulus onset asynchronies as a function of PE and difficulty levels, plotted against discrimination $d^{\prime}$. Error bars indicate standard errors of the means. Asterisks $\left({ }^{*} p<.05,{ }^{* *} p<.01\right)$ are shown only for comparisons between high and low PE levels (see the Results for the effects of difficulty level).

falling outside the RT window). An ANOVA revealed that the main effects of $\mathrm{PE}$ and difficulty levels were not significant $(p \mathrm{~s}>.10)$. The only effect of PE observed was its modulatory role on the mean RT as a function of the SOA between the grating cue and task target (Fig. 3D) [interaction between SOA and PE level: $F(2,17)=4.41, p=.029$ ]. At SOA $=400 \mathrm{~ms}$, RTs were faster for high PE than for low PE levels $(p=.022)$, whereas at SOA $=800 \mathrm{~ms}$, RTs were faster with low PE $(p=.025)$. In other words, for lowPE cues, the primary-task RT became slightly faster as SOA increased, but SOA had no such effect for highPE cues. Although this result suggests that PE modulated task set performance, it did not suggest that higher PE improved overall task set performance. 


\section{Experiment 2B}

We conducted Experiment 2B to reexamine the effect of perceptual confidence on task set preparation with another stimulus type, the dot motion stimuli. The general procedure was identical to that of Experiment 2A.

\section{Method}

Participants Twenty-two students (11 female, 11 male; mean age 24.5 years) from Columbia University participated in the same manner as in the above experiments.

Calibration, discrimination, and task set blocks The calibration and discrimination blocks followed the same procedure as in Experiment 1B except that, as in Experiment 2A, a black letter $\mathrm{X}\left(0.27^{\circ}\right.$ wide $)$ followed the dot motion stimulus with an SOA of $600 \mathrm{~ms}$.

The task set block was identical to that in Experiment 2A, with the exception that dot motion stimuli were used as the task cues, with the direction of coherent dot motion (left or right, 66-ms duration) cuing the type of upcoming task (phonological or numerical). As in Experiment 2A, a total of 60 dummy trials were randomly inserted in the block, so as to remind participants of the assigned associations between the motion direction and task type. The dummy trials contained no noise dots and had PE dots whose number was 1.5 times more than that in the high-PE/easy condition.

The performance $\left(d^{\prime}\right)$ decreased from the first block $(M=$ $1.28 \pm 0.54)$ to the second block $(M=0.89 \pm 0.58)[t(21)=$ $6.03, p<.01]$, suggesting some fatigue across blocks. Since such fatigue was not observed in the other three experiments, it is likely that the overall task demand in this experiment was relatively great, due to the general difficulty of task set preparation as well as the difficulty of discriminating the dot motion stimuli that were masked by a letter X. However, to make the results of this experiment comparable to those from the other three experiments, we collapsed the data between the blocks as in the other experiments.

\section{Results}

Discrimination blocks The means for discrimination $d^{\prime}$ and confidence ratings were calculated as in the above experiments.

The analyses of confidence rating revealed significant main effects of difficulty level $\left[F(1,21)=36.06, p<.001, \eta_{\mathrm{p}}{ }^{2}=.63\right]$ and PE level $\left[F(1,21)=18.93, p<.001, \eta_{\mathrm{p}}{ }^{2}=.47\right]$, showing that higher difficulty level as well as higher PE led to higher confidence ratings (Fig. 4A). The interaction between difficulty and PE level was nonsignificant $(p=.20)$. Thus, the manipulation of confidence was successful with motion stimuli.
However, the analyses also revealed that discrimination $d^{\prime}$ covaried with our manipulation of confidence. That is, the analysis of $d^{\prime}$ also revealed significant main effects of difficulty $\left[F(1,21)=55.41, p<.001, \eta_{\mathrm{p}}{ }^{2}=.73\right]$ and PE level $[F(1,21)$ $\left.=15.95, p<.001, \eta_{\mathrm{p}}{ }^{2}=.43\right]$. That is, higher difficulty and PE level each contributed to enhance discrimination $d^{\prime}$. These effects did not show a significant interaction $(p=.39)$. Given this, in order to examine the effect of higher confidence on task set performance without reflecting the difference in $d^{\prime}$, we eliminated data from the participants who had the largest difference in $d^{\prime}$ between the high and low PE levels (i.e., $d^{\prime}$ differences one standard error larger than the acrossparticipant mean). This eliminated a total of eight participants. With the remaining 14 participants (Fig. 4B), $d$ 's became similar between high and low PE levels $[F(1,13)=4.33, p=.06$, $\left.\eta_{\mathrm{p}}{ }^{2}=.25\right]$, whereas confidence was still significantly higher for the high than for the low PE level $[F(1,13)=11.21, p=$ $\left..005, \eta_{\mathrm{p}}{ }^{2}=.46\right]$. We analyzed task set performance using these selected participants who achieved different levels of confidence with similar levels of $d^{\prime}$. Since similar levels of $d^{\prime}$, rather than statistically matched levels of $d^{\prime}$, are sufficient to examine the effect of perceptual confidence, we analyzed the effect of PE level on task set performance with these selected participants.

Task set block The means for the cued task RTs (Fig. 4C) were calculated as in Experiment 2A. The mean RT was calculated for correct trials whose RTs were within three standard deviations of the mean $(7.4 \%$ of trials were eliminated for incorrectness, and an additional $2.2 \%$ were eliminated for falling outside the RT window). A 2 (PE level) $\times 2$ (difficulty level) repeated measures ANOVA on the selected 14 participants revealed no significant main effects or interactions $(p \mathrm{~s}>.10)$ (Fig. 4D). These results remained the same $(p s>.10)$ even when the data from all participants were analyzed (Fig. 4C). This result suggests that the higher confidence achieved with the high-PE stimuli did not enhance task set preparation. This result is in agreement with that of Experiment 2A, ensuring its robustness. Yet, whereas confidence seemed to modulate the time course of task set preparation in Experiment 2A (i.e., a significant interaction between PE level and SOA), we did not observe such an effect in this experiment. This was likely because the onset of the task cue was more ambiguous with the dynamic dot motion stimuli than with the static gratings used in Experiment 2B, diffusing the temporal effect of PE level on RTs.

\section{Discussion}

In summary, we found that performance in a go/no-go paradigm (Exps. 1A and 1B) and a task-cueing paradigm (Exps. $2 \mathrm{~A}$ and $2 \mathrm{~B}$ ) was largely explained by perceptual accuracy and 
A

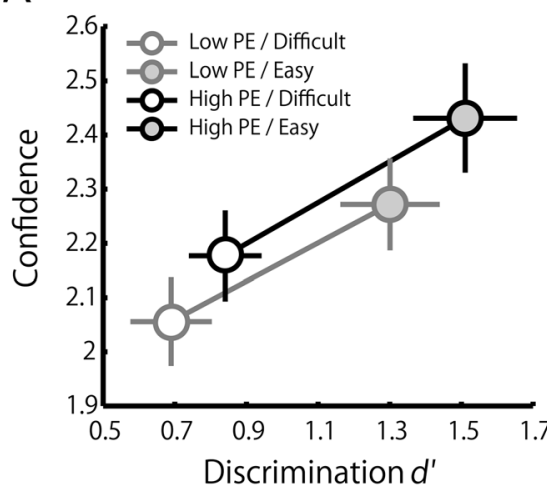

C

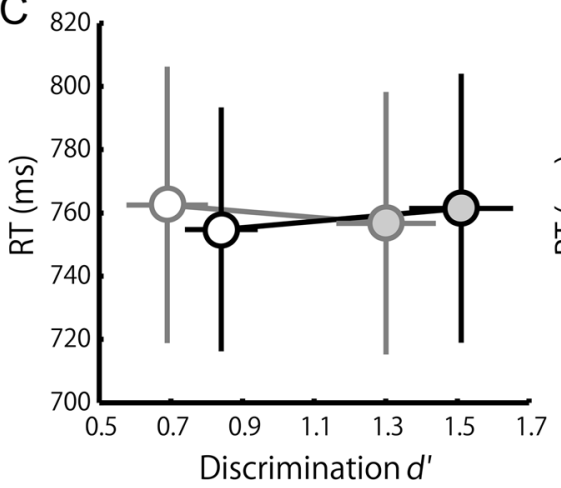

Fig. 4 Results from Experiment 2B. Discrimination confidence is plotted against $d^{\prime}$ for all 22 participants (A) and for the selected 14 participants, on the basis of their $d^{\prime}(\mathbf{B})$. Cued-task RTs (in milliseconds) plotted against $d^{\prime}$ for all participants $(\mathbf{C})$ and for the selected participants (D). Error bars

had little room to be predicted by the perceptual confidence for the stimuli utilized as go/no-go signals or task cues. We observed some effects of confidence on task performance: Higher confidence led to a more liberal response bias in the go/no-go task (Exp. 1A) and modulated the time course of task set preparation (Exp. 2A). The former result, on response bias, suggests that perceptual confidence may evoke actions in general, leading to a liberal response bias. This is in line with the reports of blindsight patients showing low perceptual confidence as well as less voluntary responses to external stimuli (i.e., conservative response criteria; Ko \& Lau, 2012). Similarly, the latter result, on the preparation time course, suggests that perceptual confidence may facilitate and increase the readiness for action toward the stimuli. However, these effects were subtle, in that they were not concerned with overall performance on the cognitive control tasks. These results seem to be robust, since they were replicated with different sets of stimuli: gratings (Exps. 1A and 2A) and dot motion (Exps. 1B and 2B).

Our behavioral finding that inhibitory control performance was similar under different levels of perceptual confidence is perhaps surprising, given that confidence may be linked to awareness (Peirce \& Jastrow, 1885), which has been suggested to be critical for cognitive control (Dehaene \&
B
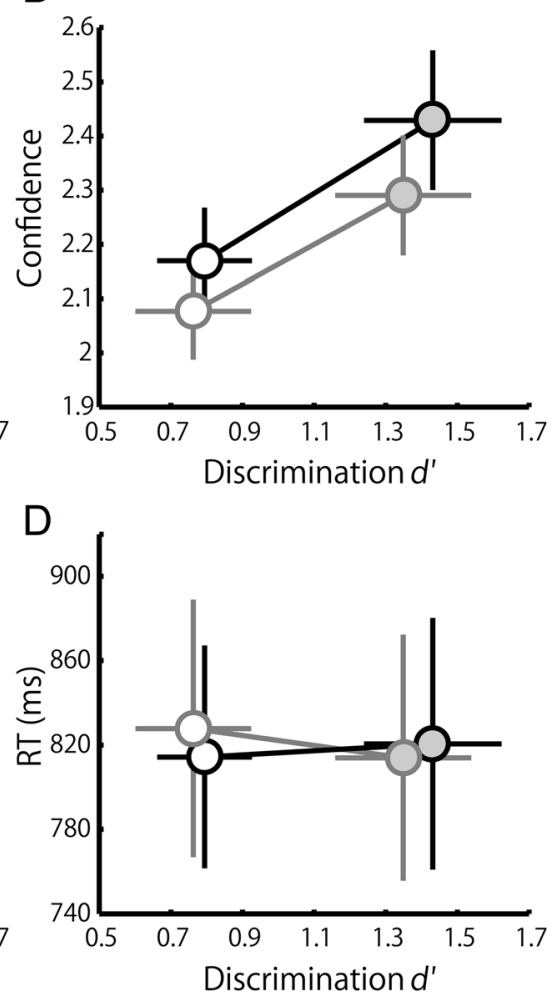

indicate standard errors of the means. Asterisks $\left({ }^{*} p<.05,{ }^{* *} p<.01\right)$ are shown only for comparisons between high and low PE levels (see the Results for the effects of difficulty level).

Naccache, 2001; Jack \& Shallice, 2001; Norman \& Shallice, 1986). Yet, other studies have shown that unconscious stimuli can trigger cognitive control (Lau \& Passingham, 2007), including response inhibition (Hughes, Velmans, \& De Fockert, 2009; Lau \& Passingham, 2007; Mattler, 2003; van Gaal, Ridderinkhof, Fahrenfort, Scholte, \& Lamme, 2008; Wokke, van Gaal, Scholte, Ridderinkhof, \& Lamme, 2011) and task set preparation (Lau \& Passingham, 2007; Mattler, 2003). Together, these results suggest that perhaps neither awareness nor subjective confidence is critical for response inhibition or task set preparation.

Likewise, perceptual confidence has been linked to subregions in prefrontal cortex, such as the dorsolateral and anterior prefrontal regions (Fleming \& Dolan, 2012; McCurdy et al., 2013), which are also known to be important for cognitive control (Badre, 2008; Koechlin \& Hyafil, 2007; Miller \& Cohen, 2001; Passingham \& Wise, 2012). Given these reports, one might expect higher perceptual confidence to result in better cognitive control. That our results did not support this prediction could be because perceptual confidence and cognitive control are housed in different subregions of the prefrontal cortex. Alternatively, it could be that whereas stimuli perceived with high confidence may recruit the prefrontal cortex to implement cognitive control, those perceived with low 
confidence may recruit alternative neural mechanisms to compensate. Future studies may directly investigate these possibilities.

Although our experimental approach in these studies was based on a manipulation of confidence, it may also find fruitful application in the study of the cognitive functions of consciousness (Dehaene \& Naccache, 2001; Jack \& Shallice, 2001; Norman \& Shallice, 1986; Lau \& Rosenthal, 2011), provided that one assumes that differences in perceptual confidence reflect differences in conscious perception. One advantage of our approach is that we did not need to experimentally abolish stimulus awareness completely to examine its functions, as is typical in subliminal-priming studies (Kouider \& Dehaene, 2007; Van den Bussche, Van den Noortgate, \& Reynvoet, 2009). The complete lack of stimulus awareness is generally difficult to achieve; it is a statistical challenge to show that any awareness measure (e.g., $d^{\prime}$ ) is exactly zero. Also, when stimulus awareness and perceptual confidence are impaired due to stimulus manipulations (e.g., masking), any effect of such stimuli is often small in size, which brings another statistical challenge in telling apart small and null effects. Even when such effects of unconscious stimuli are observed, the replication of such effects is yet another notorious problem (Shanks et al., 2013). Our approach is free from these issues, because it deals with a range of $d^{\prime}$ that is near or even above threshold levels (i.e., $d^{\prime} \approx 1-2$ ), as in typical psychophysical experiments. Even with a reasonable range of $d^{\prime}$, our method yielded robust statistical differences in confidence levels between different conditions. These advantages were discussed previously (Schmidt \& Vorberg, 2006), and we hope that our method can stimulate future efforts to understand the functions of consciousness.

Although we were generally able to manipulate confidence in all four experiments, we admit that the manipulation did not succeed in some conditions. For example, whereas the confidence manipulation was generally successful with grating stimuli (Exps. 1A and 1B), when dot motion stimuli were used (Exps. 2A and 2B), confidence covaried with $d^{\prime}$ in some participants. This suggests that certain stimulus types may be more compatible with our manipulation technique. Also, even with grating stimuli (Exp. 2A, easy condition), the mere presence of a letter stimulus after the grating seems to interfere with the confidence manipulation. With these results, future studies may further refine the methods to more robustly manipulate perceptual confidence.

One potential limitation of our experiment design particularly is that confidence was reported in the discrimination blocks but not in the cognitive control tasks (i.e., go/no-go and task set preparation). Because of this, one may argue that confidence may not have been experienced in the cognitive control tasks in the same way that it was reported in the discrimination tasks. Although we admit this possibility, we still observed some effects of perceptual confidence (e.g., criterion effect), which suggests that confidence was experienced to some extent, even though participants were not asked about it. Also, some theorists argue that perceptual certainty is intrinsically embedded in our basic perceptual processing (Ma, Beck, Latham, \& Pouget, 2006). Thus, we argue that we were, to some extent, able to manipulate perceptual confidence even in the cognitive control tasks. In our study, confidence ratings were omitted with that task because the cognitive control tasks required fast responses that could have been interfered with by the intervening confidence report. However, one recent study (Desender, Van Opstal, \& Van den Bussche, 2014) inserted a subjective rating (i.e., experienced conflict) in a go/no-go trial, which was shown to modulate conflict adaptation. Thus, future studies may strengthen the present findings by directly measuring confidence while performing cognitive control tasks.

One may also argue that the absence of a confidence effect on cognitive control was due to the relatively short duration between the onset of the cues for cognitive control (e.g., grating) and response. That is, a longer duration may have been required to observe the effect of confidence, which might have required some more time to build up. Yet this is unlikely, because we did observe some effects of perceptual confidence (e.g., a criterion effect), suggesting that our time window was sufficient to induce and observe an effect of perceptual confidence.

One important terminological issue concerns our use of the phrases positive evidence and negative evidence. Here we treated $\mathrm{PE}$ as the perceptual evidence favoring a correct stimulus identification and $\mathrm{NE}$ as the evidence favoring an incorrect identification. This usage differs from that of Zylberberg et al. (2012), in that they distinguished between the perceptual evidence favoring a chosen stimulus identification response made by a perceiver and the evidence favoring an alternative identification response. As we described in our Method section (Exp. 1A), the adoption of our definition was necessary in order for us to meaningfully manipulate $\mathrm{PE}$ and $\mathrm{NE}$ in the context of cognitive control tasks. However, future studies should pay extra attention to this terminological issue and manipulate the different kinds of PEs and NEs accordingly, with respect to the research questions.

In summary, our results suggest that perceptual confidence does not facilitate the overall effectiveness of response inhibition or task set preparation. Perhaps yet other functions of cognitive control can benefit from perceiving stimuli with higher confidence or awareness; we do not rule out such a possibility. Whatever the cognitive functions that benefit from perceptual confidence might be, our present approach provides a potentially powerful method to search for those functions.

Author note This work was supported by a grant from the Templeton Foundation (No. 21569). We thank Aaron Apple, Xiaoyu Yan, and Guy Gerard Graney for helping with the data collection. We thank Ka Yuet Liu for advice on the data analysis. 


\section{References}

Badre, D. (2008). Cognitive control, hierarchy, and the rostro-caudal organization of the frontal lobes. Trends in Cognitive Sciences, 12, 193-200. doi:10.1016/j.tics.2008.02.004

Brainard, D. H. (1997). The psychophysics toolbox. Spatial Vision, 10, 433-436. doi:10.1163/156856897X00357

Chikazoe, J., Jimura, K., Asari, T., Yamashita, K., Morimoto, H., Hirose, S., \& Konishi, S. (2009). Functional dissociation in right inferior frontal cortex during performance of go/no-go task. Cerebral Cortex, 19, 146-152. doi:10.1093/cercor/bhn065

Dehaene, S., \& Naccache, L. (2001). Towards a cognitive neuroscience of consciousness: Basic evidence and a workspace framework. Cognition, 79, 1-37. doi:10.1016/S0010-0277(00)00123-2

Del Cul, A., Dehaene, S., Reyes, P., Bravo, E., \& Slachevsky, A. (2009). Causal role of prefrontal cortex in the threshold for access to consciousness. Brain, 132, 2531-2540. doi:10.1093/brain/awp111

Desender, K., Van Opstal, F., \& Van den Bussche, E. (2014). Feeling the conflict: The crucial role of conflict experience in adaptation. Psychological Science, 25, 675-683. doi:10.1177/ 0956797613511468

Fleming, S. M., \& Dolan, R. J. (2012). The neural basis of metacognitive ability. Philosophical Transactions of the Royal Society B, 367, 1338-1349. doi:10.1098/rstb.2011.0417

Hughes, G., Velmans, M., \& De Fockert, J. (2009). Unconscious priming of a no-go response. Psychophysiology, 46, 1258-1269. doi:10. 1111/j.1469-8986.2009.00873.x

Jack, A. I., \& Shallice, T. (2001). Introspective physicalism as an approach to the science of consciousness. Cognition, 79, 161-196.

Ko, Y., \& Lau, H. (2012). A detection theoretic explanation of blindsight suggests a link between conscious perception and metacognition. Philosophical Transactions of the Royal Society B, 367, 14011411. doi: $10.1098 /$ rstb.2011.0380

Koechlin, E., \& Hyafil, A. (2007). Anterior prefrontal function and the limits of human decision-making. Science, 318, 594-598. doi:10. 1126/science. 1142995

Kouider, S., \& Dehaene, S. (2007). Levels of processing during nonconscious perception: A critical review of visual masking. Philosophical Transactions of the Royal Society B, 362, 857-875. doi:10.1098/rstb.2007.2093

Lau, H. (2009). Volition and the functions of consciousness. In M. Gazazzaniga (Ed.), The cognitive neurosciences (5th ed., pp. 1191-1200). Cambridge, MA: MIT Press.

Lau, H., \& Passingham, R. E. (2006). Relative blindsight in normal observers and the neural correlate of visual consciousness. Proceedings of the National Academy of Sciences, 103, 18763 18768. doi:10.1073/pnas.0607716103

Lau, H., \& Passingham, R. E. (2007). Unconscious activation of the cognitive control system in the human prefrontal cortex. Journal of Neuroscience, 27, 5805-5811. doi:10.1523/JNEUROSCI. 433506.2007

Lau H, Rosenthal D. (2011) Empirical support for higher-order theories ofconscious awareness. Trends in Cognitive Sciences 15(8):365-73. doi:10.1016/j.tics.2011.05.009

Ma, W. J., Beck, J. M., Latham, P. E., \& Pouget, A. (2006). Bayesian inference with probabilistic population codes. Nature Neuroscience, 9, 1432-1438. doi:10.1038/nn1790

Macmillan, N. A., \& Creelman, C. D. (2005). Detection theory: A user's guide. Mahwah, NJ: Erlbaum.
Maniscalco, B., \& Lau, H. (2012). A signal detection theoretic approach for estimating metacognitive sensitivity from confidence ratings. Consciousness and Cognition, 21, 422-430. doi:10.1016/j.concog. 2011.09.021

Mattler, U. (2003). Priming of mental operations by masked stimuli. Perception \& Psychophysics, 65, 167-187. doi:10.3758/ BF03194793

McCurdy, L. Y., Maniscalco, B., Metcalfe, J., Liu, K. Y., de Lange, F. P., \& Lau, H. (2013). Anatomical coupling between distinct metacognitive systems for memory and visual perception. Journal of Neuroscience, 33, 1897-1906. doi:10.1523/JNEUROSCI. 189012.2013

Michelson, A. (1927). Studies in optics. Chicago, IL: University of Chicago Press.

Miller, E. K., \& Cohen, J. D. (2001). An integrative theory of prefrontal cortex function. Annual Review of Neuroscience, 24, 167-202. doi: 10.1146/annurev.neuro.24.1.167

Norman, D. A., \& Shallice, T. (1986). Attention to action: Willed and automatic control of behavior. In R. J. Davidson, G. E. Schwartz, \& D. Shapiro (Eds.), Consciousness and self-regulation: Advances in research (Vol. 4, pp. 1-18). New York, NY: Plenum Press.

Passingham, R. E., \& Wise, S. P. (2012). The neurobiology of the prefrontal cortex: Anatomy, evolution, and the origin of insight. New York, NY: Oxford University Press.

Peirce, C. S., \& Jastrow, J. (1885). On small differences in sensation. Memoirs of the National Academy of Sciences, 3, 73-83.

Rounis, E., Maniscalco, B., Rothwell, J. C., Passingham, R. E., \& Lau, H. (2010). Theta-burst transcranial magnetic stimulation to the prefrontal cortex impairs metacognitive visual awareness. Cognitive Neuroscience, 1, 165-175. doi:10.1080/17588921003632529

Schmidt, T., \& Vorberg, D. (2006). Criteria for unconscious cognition: Three types of dissociation. Perception \& Psychophysics, 68, 489504. doi:10.3758/BF03193692

Shanks, D. R., Newell, B. R., Lee, E. H., Balakrishnan, D., Ekelund, L., Cenac, Z., \& Moore, C. (2013). Priming intelligent behavior: An elusive phenomenon. PLoS One, 8, e56515. doi:10.1371/journal. pone. 0056515

Van den Bussche, E., Van den Noortgate, W., \& Reynvoet, B. (2009). Mechanisms of masked priming: A meta-analysis. Psychological Bulletin, 135, 452-477. doi:10.1037/a0015329

van Gaal, S., Ridderinkhof, K. R., Fahrenfort, J. J., Scholte, H. S., \& Lamme, V. A. F. (2008). Frontal cortex mediates unconsciously triggered inhibitory control. Journal of Neuroscience, 28, 80538062. doi:10.1523/JNEUROSCI. 1278-08.2008

van Gaal, S., Ridderinkhof, K. R., Scholte, H. S., \& Lamme, V. A. F. (2010). Unconscious activation of the prefrontal no-go network. Journal of Neuroscience, 30, 4143-4150. doi:10.1523/ JNEUROSCI. 2992-09.2010

Watson, A. B., \& Pelli, D. G. (1983). QUEST: A Bayesian adaptive psychometric method. Perception \& Psychophysics, 33, 113-120. doi:10.3758/BF03202828

Weiskrantz, L. (2009). Blindsight: A case study spanning 35 years and new developments. Oxford, UK: Oxford University Press.

Wokke, M. E., van Gaal, S., Scholte, H. S., Ridderinkhof, K. R., \& Lamme, V. A. F. (2011). The flexible nature of unconscious cognition. PLoS One, 6, e25729. doi:10.1371/journal. pone. 0025729

Zylberberg, A., Barttfeld, P., \& Sigman, M. (2012). The construction of confidence in a perceptual decision. Frontiers in Integrative Neuroscience, 6, 79. doi:10.3389/fnint.2012.00079 